\title{
Appropriate Technology
}

\section{Ophthalmology in developing countries}

\author{
JOHN SANDFORD-SMITH
}

In the West ophthalmology is sometimes considered to be a minor specialty, but in developing countries it is one of the most important because the prevalence of blindness in many rural areas ranges from 1 to even $5 \%$, compared with about $0.1 \%$ in most Western countries. Ophthalmological disorders represent not only a larger problem but also a different one: in developed countries the most common cause of blindness is degenerative or vascular disorders of the retina while in the developing countries five major diseases are responsible for most of the blindness - namely, trachoma, xerophthalmia, cataract, onchocerciasis, and glaucoma.

\section{Major causes of blindness}

Trachoma is common throughout the tropics but especially where there is a combination of unhygienic living conditions, a dry dusty atmosphere, and flies. At least two million people are blinded by this disorder, and it causes discomfort and partial visual loss in about another hundred million. ${ }^{1}$ The trachoma organism is spread from eye to eye, usually in young children, by close contact and particularly by flies. Trachoma may be a recurrent disease because one attack confers only a low level of immunity. Recurrent episodes cause increasingly severe conjunctival scarring. Poor hygiene also predisposes towards a high incidence of other types of conjunctivitis, especially adenovirus and bacterial conjunctivitis, which further increase the severity of the conjunctival and corneal scarring. The most effective preventive measure is to promote good hygiene to decrease the fly population and lessen the risk of direct eye to eye spread by contact in young children. Intermittent use of tetracycline eye ointment is also helpful both for treatment and to control the spread of the disease in the community by cutting down the number of active carriers. Tetracycline ointment twice daily for five days each month for six months is the schedule recommended by the World Health Organisation.

Nutritional corneal ulceration is one of the most distressing causes of blindness because it affects primarily young and underprivileged children. In Asia alone it afflicts at least one hundred thousand children each year. ${ }^{3}$ The causes are complex-the most important are a dietary deficiency of vitamin A and measles. Vitamin A intake may be increased by nutritional advice, by fortification of foods, or by giving capsules of vitamin A every six months. Measles may be prevented by vaccination.

Cataract occurs in all communities but is thought to develop at a younger age in hot countries. About three million people in Africa and five million people in India are blind as a result of cataracts. ${ }^{\prime} \mathrm{O}$ the five major causes of blindness, this is the only one that is treatable. Prevention is at present impossible, but simple eye surgery should restore useful sight.

Onchocerciasis or river blindness due to the Onchocerca volvulus

Leicester Royal Infirmary, Leicester LE1 5WW

JOHN SANDFORD-SMITH, FRCS, consultant ophthalmologist worm is found in the Savannah region of central Africa, with pockets of infection in central America and Yemen. In many villages its effects are devastating. Blindness usually develops between the ages of 20 and 40 . Prevention may be achieved by controlling the insect vector, and the World Health Organisation (working in the Volta river basin in West Africa) is using insecticide spray to control the similium fly larvae, which are found in small fast flowing streams and rivers. It plans to keep this up for about 20 years because that is the estimated life cycle of the worm.

Glaucoma occurs in all communities and is an increasingly important cause of blindness with advancing age. Acute glaucoma is rare in negroes, but they are more susceptible to chronic glaucoma. The only realistic way of detecting early glaucoma that I can think of is to have facilities for eye examination and tonometry available throughout the community. This is obviously a counsel of perfection, but wherever appropriately trained primary health care workers or ophthalmic nurses are active in the community they should be able to increase the detection of primary glaucoma in its early stages.

\section{Problems in practising prevention of blindness}

Another important feature of ophthalmology in developing countries is the variety of eye disease in different areas. Variations in climate, insect vectors, social surroundings, and nutrition profoundly affect the pattern of eye disease. For example, in Africa on the edge of the Sahara Desert dust, glare, and flies are associated with a high incidence of blindness from trachoma, and corneal conditions such as pterygium and solar keratopathy. Further south in the Savannah dust and flies become less of a problem and the incidence of trachoma falls. This, however, is the zone for onchocerciasis, which in some areas may devastate the entire community. Further south still, in the tropical rain forest, diseases like that due to Loa-loa, which causes acute oedema of the eyelid, and nutritional optic atrophy from cassava are found. Throughout Africa there is a high incidence of measles, corneal ulcers, vernal conjunctivis (chronic allergic conjunctivitis), and leprosy affecting the eye.

To confront this vast and varied problem of eye disease with limited resources and a shortage of skilled staff, not only different technology but a different philosophy of what ophthalmology is all about is needed. Western ophthalmology is mainly concerned with curative treatment, and the equipment used in diagnosis and surgical treatment is complex and expensive. In developing countries prevention is all important, and although Western postgraduate training and degrees are highly valued such training is not suited to tackling the major blinding diseases in rural communities. Most doctors who have obtained their ophthalmological training in the West go into clinical practice in the big cities and have little contact with the poor rural areas. The people who live there (who make up most of the blind people) are, therefore, poorly served, and for most their only treatment is from unqualified, traditional healers. Although the World Health Organisation is encouraging cooperation with traditional healers in many branches of medicine, this is not advisable in ophthalmology, for unskilled interference is of limited value and may be disastrous (figs 1, 2). 


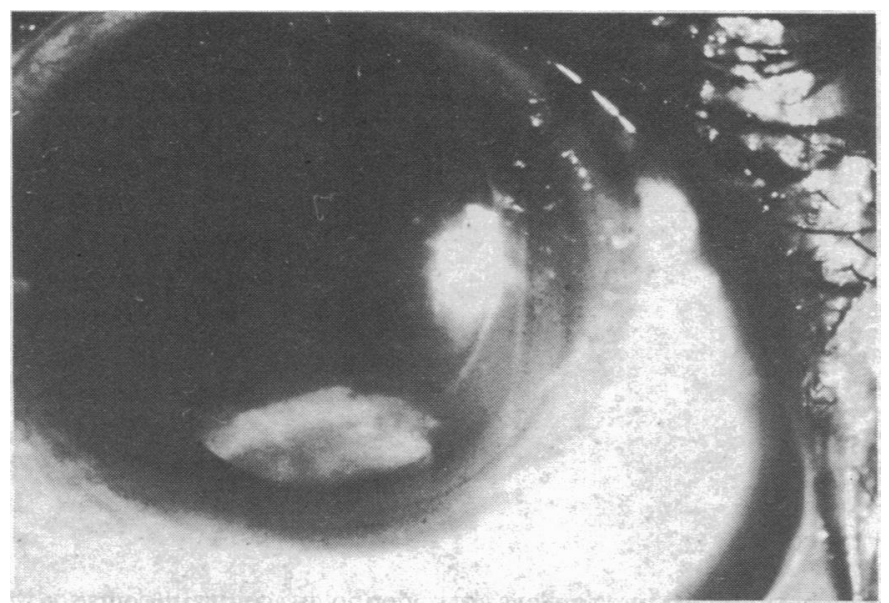

FIG 1-An eye after couching for cataract. Itinerant couchers are still active in some parts of the world. A needle is inserted at the limbus and the lens pushed into the vitreous. The opaque lens can just be seen, through the dilated pupil lying in the lower half of the vitreous. Unfortunately blindness from uveitis or endophthalmitis is the uşual result.

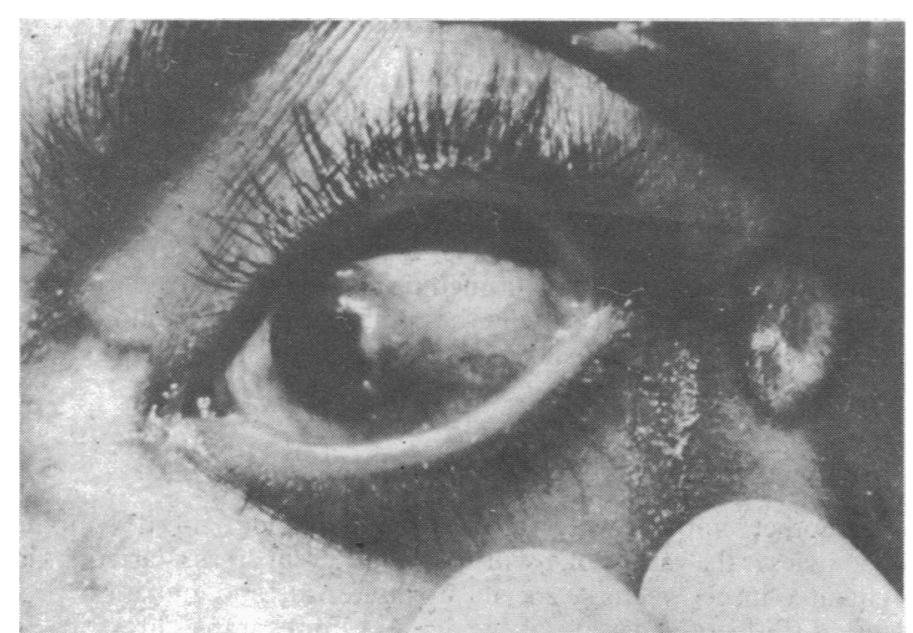

FIG 2-A patient with vernal conjunctivitis. Cautery has been applied to the skin at the inner and outer canthus.

What, then, is needed to provide an adequate ophthalmic service in the Third World? Not lots of money, not lots of equipment, not even lots of specialists packed like sardines in the middle class areas of the big cities, but a service that is oriented towards the rural areas and prevention. This may be achieved with limited resources and equipment, provided that it is realistically planned and carried out by dedicated workers. There must be good two way contact between the community and the eye specialist. The best way to achieve this is to train primary health care workers or ophthalmic nurses to make the initial contact and then liaise with the ophthalmologist. Village and community leaders may be taught how to prevent blindness from diseases like trachoma, vitamin A deficiency, and measles. Other specialists may be enlisted to help-for example, horticulturists can identify foods rich in vitamin A that may be grown locally. Thus eye specialists must be encouraged to spend some of their time training and supervising medical assistants, nurses, dispensers, and primary health care workers. Obviously most specialists will want a base in a major city, but ophthalmology, with its easily portable equipment, lends itself to mobile work. It is much easier for one ophthalmologist and one trained nurse to travel 200 miles than for a hundred bewildered, blind, and elderly patients with their escorts to travel in the opposite direction.

There are encouraging signs that a more community oriented type of ophthalmic care is being provided in some countries-for example, the mobile eye hospitals, eye camps, and eye safaris in the
Indian subcontinent and in some parts of Africa. These are sponsored by the government or by voluntary agencies and offer specialist eye care to the rural communities. The type of service that they usually offer is screening all the local population who complain of problems with their eyes. Those who need medical treatment or glasses are, whenever possible, given them, and those who need simple surgery -for example, for correction of trachomatous entropion, glaucoma, or removal of cataracts-may have that surgery performed locally. Cataracts may be removed very cheaply by a small surgical team: usually a nurse prepares the patient and gives a local anaesthetic, cleans the face and eye, and applies sterile drapes. Ideally, the surgical team has more than one operating table and the surgeon can go from table to table doing simple cataract extractions. This entails making a limbal incision in the eye, a broad iridectomy, removal of the lens, and putting in three or four corneal scleral sutures. In this way a team of two doctors, six to eight nurses, and various unskilled orderlies may get through 100 operations a day. A patient may have his cataract removed (including the price of the drugs and dressings) and be given a pair of spectacles to restore his vision to normal for about US\$10 (fig 3).

More complex surgical problems-for example, retinal detachments and corneal grafting - are not usually carried out under such circumstances. In some cases the mobile eye clinic provides only a diagnostic service and medical treatment. Patients who need surgery are identified and sent elsewhere.

\section{Equipment}

Snellen's chart for testing visual acuity and a pin hole test that distinguishes refractive errors from eye diseases are essential. A black screen for testing visual fields is helpful, but a simple confrontation test using one's own fingers will detect serious field defects.

An ophthalmoscope is essential. Many people do not realise that an ophthalmoscope may also be used to examine the front of the eye

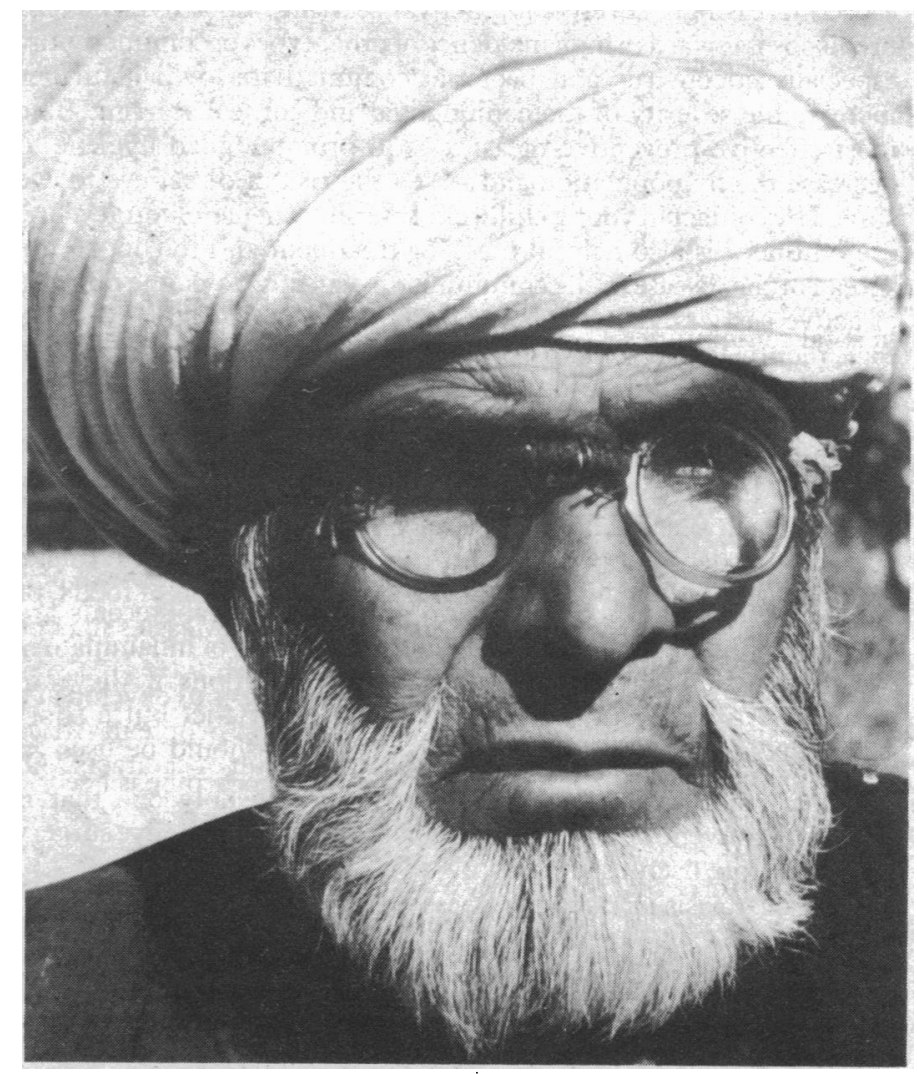

FIG 3-A grateful patient some years after a cataract operation with a battered but functional pair of glasses. 
as well as the fundus. By holding the instrument as for fundus examination but using a strong positive lens (about +15 or +20 ) a magnified and illuminated view of the front of the eye is obtained. If the pupil is dilated slight opacities in the cornea, lens, or vitreous may be seen standing out against the red fundus reflex. A simple torch light and magnifying lens are also helpful for examining the cornea and conjunctiva, and fluorescein paper is essential for detecting corneal ulcers. A tonometer for measuring intraocular pressure is another essential item. The old fashioned Schiotz tonometer is cheap, and if kept scrupulously clean and used correctly it is reliable. Applanation tonometers give more consistent results; the Perkins tonometer and the Glaucotest (made in Germany by Heine) are two useful examples.

A simple microscope to examine skin snips for microfilaria is important in areas where onchocerciasis is endemic. More expensive items include a slit lamp and a binocular indirect ophthalmoscope (for example, the Schultz Crock, which has rechargeable batteries, costs $£ 500$ ). These are expensive but provide a superb view of the front and back of the eye respectively and are essential diagnostic tools for the specialist working in a major eye centre.

\section{Surgery}

Nearly all surgery on adults may be done under local anaesthetic. Ophthalmic surgical instruments need to be of good quality and should be packed, handled, and sterilised with great care. Some magnification is necessary for most eye surgery, but an operating microscope is an expensive luxury and perfectly adequate magnification for all but the finest procedures may be achieved using a telescopic pair of operating glasses that will magnify from $\times 2$ to $\times 4$. Of these I recommend those made by Zeiss (East Germany), which cost about $£ 80$. Most of the instruments described are robust and, provided the batteries are not left to run down and corrode (and instruments are used for which local batteries and a good supply of bulbs are available), there should be no great problem in operating them. Slit lamps may be run off car batteries with appropriate connections if they are used by mobile eye teams.

Cataract surgery is the most common operation, but other important procedures include the correction of trachomatous entropion and the treatment of glaucoma by iridectomy or a drainage operation. Ideally, only a trained specialist should perform eye surgery of any sort, but there is such a shortage of trained ophthalmologists in many developing countries that either a general duties doctor or a nurse with specific ophthalmological training may be taught some of the basic surgical skills. They should then be able to carry out safely the three procedures mentioned above, which encompass up to $90 \%$ of the potentially treatable blinding conditions in rural communities.

\section{Drugs}

Basic ophthalmology does not require many drugs, and most drops and ointments may be made up by the pharmacist. Tetracycline ointment is the best local treatment for trachoma and chloroamphenicol a good all round antibiotic. Local steroids are effective in the treatment of uveitis and other forms of inflammatory eye disease but may result in serious complications if they are misused. Atropine is the best long acting mydriatic, but a short acting mydriatic - for example, homatropine - should be used to dilate the pupil before examining the fundus. Pilocarpine is needed for patients with glaucoma, to prepare the eye for surgery. Medical treatment of glaucoma is usually inappropriate because the patients cannot afford the drugs and are unlikely to comply with long term regular treatment. Many antibiotics and steroid preparations may be injected subconjunctivally to treat severe infection or inflammation of the eye. Gentamicin is particularly useful as a broad spectrum injectable antibiotic for bacterial corneal ulcers or endophthalmitis. A supply of placebo drops (zinc sulphate or saline) is useful in some countries where the prevailing opinion is that "a doctor who doesn't prescribe any medicine can't be any good."

\section{Spectacles}

In developing countries facilities for manufacturing and fitting spectacles vary widely but spectacles can usually be manufactured locally. Two main types of glasses are needed: spectacles for near vision for elderly presbyopic patients with a power of about $+2 \cdot 5$ diopters and aphakic glasses for patients who have had cataracts removed, with a power of about +10 diopters (fig 3 ).

In conclusion, the ophthalmic services in common with other medical services in the Third World are often inadequate. The unique challenge of ophthalmology is that most blindness is avoidable without enormous expenditure. The obstacles to prevention are mostly human-unwillingness to work and travel in rural areas, fear of teaching surgical skills to non-specialists and uninterest in community health and preventive medicine-and these must be overcome.

The Institute of Ophthalmology in London holds a six month course on the prevention of blindness in developing countries. The course is organised for doctors, nurses, and administrators and is particularly concerned to teach the very varied skills needed to provide comprehensive eye care.

\section{References} 1 International Agency for the Prevention of Blindness, eds. World blindness and its prevention.
Oxford: Oxford University Press, 1980 .

2 World Health Organisation. Field methods for the control of trachoma. Geneva: WHO, 1973.

.

\section{Further reading}

Jones BR. The prevention of blindness from trachomas. Transactions of the Ophthalmological Society of the UK 1974;95:16-33.

Somer A. Field guide to the detection and contact of xerophthalmia. Geneva: WHO, 1978.

Anderson J, Fuglsang H. Ocular onchocerciasis. Trop Dis Bull 1977;74:4.

World Health Organisation. Symptomology, pathology and diagnosis of onchocerciasis. Geneva: WHO, 1974.

A patient who is a keen body builder wishes to use anabolic steroids to improve his physique. Is this wise?

The use of anabolic steroids to increase muscle bulk and strength has been criticised and condemned by many physicians, researchers, and athletic governing bodies. ${ }^{1}$ Evidence as to their efficacy is inconclusive, since their use has apparently resulted in increases in strength, body weight, and lean body mass in some studies but not in others. Testimony by individual athletes, however, suggests that many athletes use much higher doses than those evaluated in published research. Apart from differing drug dosages, other confounding factors in the interpretation of trials of these drugs include differences in individual steroids used, routes of administration, associated exercise regimens, and diet. The increase in lean body mass that may follow the use of such drugs does not seem to be due to an increase in normal muscle tissue. ${ }^{2}$ Some researchers have suggested that their mode of action is due not to an anabolic effect as such, but to the fact that they permit individuals to train at or near maximum capacity for longer periods.

Anabolic steroids have several adverse effects, which include abnormalities of liver function and, in the case of the orally active C17 alkylated drugs, a dose dependent cholestatic jaundice. There have been several reports of hepatoma associated with long standing ingestion of androgens and anabolic steroids but no cases have yet, to my knowledge, been described in athletes. All anabolic steroids have androgenic actions and, in addition to causing virilisation in women, may depress gonadal function in men. A recent Finnish study showed complete suppression of spermatogenesis within three months of starting anabolic steroids even in modest dosage and a $28 \%$ reduction in testicular size over the six month period of taking the drug. ${ }^{3}$ Most, but not all, of the athletes showed a slow recovery of sperm production during the four month detraining period, during which no further drugs were taken. Because of doubt as to their efficacy and because of these adverse effects the use of anabolic steroids in body building cannot be recommended.- LILIAN E MURCHISON, consultant physician, Aberdeen.

\footnotetext{
' American College of Sports Medicine. Position statement on the use and abuse of anabolic-androgenic steroids in sports. Med Sci Spotts 1977;9:XI-XIII. 2 Hervey GR, Knibbs AV, Burkinshaw L, et al. Effects of methandienone on the performance and body composition of men undergoing athletic training. Clin Sci Maughan RJ. Adverse effects of drug abuse in male athletes. Int $\mathcal{F}$ Sports Med 1983;
suppl:21.
} 